\title{
Emergent Semantic Patterns In Large Scale Image Dataset: A Datamining Approach
}

\author{
Umair Mateen Khan \\ Computer Science Department \\ Otago University \\ Dunedin, New Zealand \\ Email: umairkhan@cs.otago.ac.nz
}

\author{
Brendan McCane \\ Computer Science Department \\ Otago University \\ Dunedin, New Zealand \\ Email: mccane@cs.otago.ac.nz
}

\author{
Andrew Trotman \\ Computer Science Department \\ Otago University \\ Dunedin, New Zealand \\ Email: andrew@cs.otago.ac.nz
}

\begin{abstract}
In this paper we investigate an unsupervised learning method applied to low level image features extracted from a large collection of images using data mining strategies. The mining process resulted in several interesting emergent semantic patterns. Initially, local image features are extracted using image processing techniques which are then clustered to generate a bag of words $(\mathrm{BoW})$ for each image. These bags of words are then used for mining co-occurring patterns. The generated patterns were either global in nature i.e showed a behavior spread across many images or a local and more rare behavior found across few images. These patterns are assigned semantic names to build a semantic relationship among images containing them.
\end{abstract}

\section{INTRODUCTION}

There has recently been a strong interest in the learning of low-level features from training data, with a focus on neurally inspired architectures [1], [2], [3], [4]. At the same time, the number of large image databases has increased [5], [6]. In this paper we hypothesize that investigating very large image databases will result in the emergence of interesting patterns in a bottom-up sense. However, we do not start at the lowest possible level (pixels), and instead start at an intermediate level (SIFT features [7]), and investigate the co-occurrence of such features to see if more semantic level features emerge. We present an approach that explores emergent patterns in a large image collection by using both frequent and rare itemset mining strategies.

\section{Related Work}

Several significant works have looked at the application of data-mining techniques for image retrieval and object recognition applications. The most common method used is association rule mining. Association rule mining is a technique for discovering both global and local knowledge from large collections of data [8]. Data mining strategies like Apriori [9], Eclat [10] and Fp-Growth [11] have been used for finding correlations among data elements and extracting hidden relationships that are not so obvious. These techniques can be applied to any field that produces or deals with a large amount of data. Some of the applications of data mining include market basket data analysis, patient disease symptoms analysis, stock analysis and social network analysis. Similarly image mining is no exception and deals with discovering hidden relationships from image data elements that are pixels, shapes, textures or higher level features such as SIFT [7]. For all the above mentioned mining techniques and many others it is necessary for the data to be represented as market basket transactions [12] in which each transaction can contain 1 or multiple items.

Association mining among elements of data is a highly researched area but rather less studied in the context of image mining [13]. In [14] an attempt was made to mine relationships among objects from different modalities of multimedia data. These objects were called perceptual objects and were defined in a spatio-temporal window and the generated rules were used to define the relationships in a more compact and semantic way. Another approach to mine frequently co-occurring objects/actors or scenes in video frames was performed in [15]. In their approach they created a transaction for each visual word and all its neighboring words were taken as its items. Once these neighborhoods are defined they are used for mining cooccurring objects or actors among many frames. Association rule mining has also been used for clustering web images [16]. The authors first generated rules using both visual and text features obtained from web pages and then used them to generate hyper-graphs. Then a hyper-graph partitioning algorithm was used to get clusters. In [13] association rule mining was performed on regions of interest (ROI) in CT images of brains. The ROI were first extracted using a region extraction and clustering algorithm which also used domain knowledge. The rules were then generated on mined frequent itemsets considering ROI of brain images as items. In another approach [17], knowledge discovery from image contents without using any domain knowledge was investigated. Their work was based on finding relationships in basic geometric shapes but was performed on a very limited number of images. They also suggested the need for some reliable domain knowledge for better object recognition. Object class recognition was also done using association rule mining techniques in [18]. The association rules were built on low level features occurring within a bounding box containing either a background image example or an object image from one of its classes. Visual words inside this box were represented as transactions and then association rules were mined. The transaction database contained a combined set of transactions obtained from both objects and background bounding boxes. The learned rules 
were then used to tell the presence of a particular object class or a background in the unseen images. A similar approach was presented in [19] where they used it for detecting logos of different categories in an image. They achieved this by locating dense configurations of frequent local features related to each logo class. They extracted association rules on a spatial pyramid of each base feature where a base feature is represented as a group of all neighboring features inside a radius grid across it. Each base feature was then represented as a transaction and all surrounding features as items of the transaction. Human action classification by mining association rules was performed in [20]. The key idea was in the concept of compound features. Compound features were defined as groups of corner descriptors used to encode local features in space and time. These features were learned using data mining techniques by looking at their co-occurrences. The classifier was actually a group of these computed features and was capable of both recognizing and localizing a real time activity.

In all previous work the focus of using data mining techniques on images has been on a specific application. Those applications include: object recognition; object classification; clustering; scene recognition; or content-based image retrieval. The purpose of the work presented here is quite different, although the techniques used are similar. Our goal is to show that semantic level features of images can emerge in a bottomup process from a suitably large collection of images, and that these semantic features are interesting in themselves. Our long term goal is to show that the notion of semantic-ness is crucially dependent on the low-level processing of a particular vision system (natural or artificial) and that different vision systems will give rise to different notions of "semantic" and will necessarily interpret the world in different, but equally valid, ways.

\section{Feature Extraction and Clustering}

The current generation of object recognition, object classification and image retrieval systems identify certain interest points, called features using an image processing technique such as SIFT [7] or SURF [21]. To extract these features, techniques such as edge detection, corner detection [22], blob [23] and ridge detection [24] etc. are used. In this paper SIFT [7] is used to identify keypoints and the 128 dimensional SIFT descriptor is used to represent the keypoint. This descriptor contains orientation information obtained from a $4 \times 4$ grid across each keypoint. SIFT features are highly distinctive and provide invariance to different transformations e.g. rotation, translation and scaling and partial invariance to illumination and viewpoint change. SIFT has been extensively used in many object recognition and image retrieval applications [7], [18], [25], [26], [27], [28]. SIFT produces a large number of features and may generate 1000 to 2000 features per image depending on image content and size. SIFT features are clustered into a finite set of visual words using approximate K-means clustering as done in [26], [27]. For fast lookup of nearest clusters a Kd-Tree [29] is built on cluster centers. For clustering we partitioned the data into chunks so that it can be loaded in memory. Each image is represented using a visual bag of words [26] representation and the co-occurrence relationships between the words are mined to extract interesting features as outlined in the following section.

\section{Frequent Itemsets And Association Rules MINING}

Association rule mining is a technique used for mining interesting relationships in market basket transaction data. A market basket is a list of items that were purchased together by a customer. The association found is not influenced by the inherent property of the data but rather the co-occurrences in data items. Association rule mining was first suggested in [12].

Let $I=\left\{i_{1}, i_{2}, i_{3}, \ldots . i_{n}\right\}$ be the set of all possible items in the data collection (all possible visual words in our case). Let $T$ be a transaction containing a subset of these items such that $T \subseteq I$. In our case, $T$ contains all visual words from a single image. $D$ is the collection of all transactions. An association rule is an implication, $X \Rightarrow Y$, where $X \subset I$ and $Y \subset I$ but $X \cap Y=\varnothing$ [12]. Association rules have two parameters called support $s$ and confidence $c$. Support is a count of transactions in which a particular item is found. So the support of an association rule $X \Rightarrow Y$ which contains two items would be the ratio of transactions that contains $X \cup Y$ compared to the total number of transactions. Confidence is the ratio of the number of transactions that contain $X \cup Y$ to the total number of transactions that contain $X$. An association rule only holds if it has a support $s$ greater than minsup and confidence $c$ greater than minconf; values which are specified by the user.

Apart from the minconf and minsup values a further measure called lift can also be used to check the strength of a rule. The lift value of a rule tells the degree to which $Y$ is more likely to occur when $X$ occurs. A value of lift less than 1.0 means that $Y$ is less likely to occur with $X$ than $Y$ 's total support in the entire transaction dataset. A lift value higher than 1.0 implies a positive association between $X$ and $Y$. A positive association means that $X$ and $Y$ co-occur together more than expected.

The support of a rule can be defined as:

$$
\begin{aligned}
& \text { support }(X \Rightarrow Y)= \\
& \frac{\#_{-} \text {transactions_containing_both_X_and_Y }}{\text { total_\#_of_transactions }}
\end{aligned}
$$

The confidence for the rule can be calculated as:

$$
\begin{aligned}
& \text { confidence }(X \Rightarrow Y)= \\
& \frac{\#-\text { transactions_containing_both } X_{-} X_{-} a n d_{-} Y}{\#_{-} \text {of_transactions_containing_X }}
\end{aligned}
$$

And the Lift value of a rule can be defined as:

$$
\operatorname{lift}(X \Rightarrow Y)=\frac{\text { confidence }(X \Rightarrow Y)}{\text { support }(Y)}
$$

The process of association rule generation is a two stage process. First frequently co-occurring items called itemsets 
are mined using a minsup threshold. An itemset containing ' $\mathrm{r}$ ' items is called an r-itemset. Now association rules are generated from these itemsets and minconf and lift thresholds are checked. The current BOW representation of all images can be converted to market basket transactions by seeing each image as a transaction and words in that image as items of that transaction. Co-occurrence among these transactions can be found using any such association rule mining algorithm. We used the Eclat [10] algorithm for mining these co-occurring itemsets or frequent itemsets from image transactions. Although $F p$-Growth [11] yielded much better results to Eclat as described in [30], we used Eclat because in our case Fp-Tree did not fit in memory. Once we have the generated itemsets we then generated the association rules and only those rules are considered which met the minconf and lift criteria.

\section{Rare Itemsets and Association Rules Mining}

Mining frequent patterns from the data is crucial and it gives a global insight into the data. But in some cases that global insight can be easily predicted by domain experts and hence does not necessarily give useful knowledge. For example if we look at the records of patient history for a fatal disease then common symptoms can be easily mined and most of them would likely be already known to domain experts. In that case a more interesting finding would be to see which symptoms occurred rarely or were infrequent but had very high confidence. A frequent itemset miner completely ignores such itemsets because they occur in very few transactions.

An obvious way to find such rare co-occurrences is to reduce the minsup to a very low value and then a frequent itemset miner will consider these rare occurrences as frequent. This results in a very high running time of the algorithm and too many itemsets fulfilling the minsup threshold, this phenomena is known as the rare itemset problem [31]. The algorithms that are designed to mine rare itemsets use different notions of such thresholds.

For our problem we used the RP-Tree [31] algorithm that extracts rare patterns by building a prefix tree only on those transactions that contain a rare item. The algorithm is a modification of the Fp-Growth [11] algorithm. Two thresholds are used for mining rare itemsets, the first called minRareSup, is a minimum support for an item to be a rare item and works as a noise filter. All those items having support less than this threshold are not considered further. The second threshold minFreqSup is a maximum support for an itemset to be considered as a rare itemset [31]. All items having support greater than this threshold are considered frequent.

Itemsets are categorized into three different types [31]: the first is rare itemset and it includes all those itemsets that have support less than minFreqSup but greater than or equal to minRareSup. The rare-item itemsets consists of those itemsets that have both rare and frequent items but the itemset itself is rare and therefore it also fulfills the criteria for a rare itemset. The third type is non-rare-item itemset and consists of all the items which are frequent but the itemset itself is rare. We are mainly concerned with first two types of itemsets because nonrare-item itemsets are very likely to occur by chance in large datasets.

Consider the itemset $X$. It is called a rare itemset iff

$$
\begin{aligned}
& \operatorname{support}(X)<\operatorname{minFreqSup,} \\
& \text { support }(X) \geq \operatorname{minRareSup}
\end{aligned}
$$

$X$ is called rare-item itemset iff

$$
\begin{aligned}
\exists x \in X, \text { support }(x)< & \text { minFreqSup, } \\
& \text { support }(X)<\operatorname{minFreqSup}
\end{aligned}
$$

$X$ is called a non-rare-item itemset iff

$$
\begin{aligned}
& \forall x \in X, \operatorname{support}(x) \geq \operatorname{minFreqSup}, \\
& \text { support }(X)<\text { minFreqSup }
\end{aligned}
$$

\section{EXPERIMENTAL Setup AND Results}

The focus of this research is to investigate emergent patterns from a large image collection. The MIRFLICKR [32] collection containing 1 million images was downloaded. We used half of the images because we considered it to be sufficient for our experiments. The dataset contains high quality images of everyday scenes and is designed for image retrieval applications. It has been used in ImageCLEF [6] evaluation forum for the last 3 years. Some of the images from this collection are shown in Figure 1. The dataset was suited to our scenario because we are learning unsupervised, and do not want to use a dataset containing many similar scenes. For feature extraction, SIFT [7] was used and more then 10 billion local features were extracted. Features from each image were saved in separate files along with their $\mathrm{X}$ and $\mathrm{Y}$ coordinates. All experiments were performed on 64-bit Intel Core 2 Duo $3.00 \mathrm{GHz}$ CPU with internal memory of 4 GB.

Approximate k-means clustering was then performed on all the features and an image chunk size of 10,000 images was chosen because that was the largest chunk size that could consistently fit all of the features in memory. For one iteration of the clustering process features from 50 chunks were processed i.e a total 500,000 images. The clustering process was terminated after 10 such iterations and the clustering took about 12 hours.

The selection of $k$ (total cluster centers) was very important and the most crucial in our case as a wrong value of $k$ can greatly effect the mining process by increasing or decreasing the co-occurrences. A large value of $k$ can increase false negatives as most features will match to different clusters even though there would be only slight differences. On the other hand a small value of $k$ will increase false positives as many features that are different from each other will match the same cluster center. As we were unsure about the correct number of clusters we decided to use five different values of $k$. These values were set to 5,000, 15,000, 35,000, 50,000 and 75,000 respectively. Once these clusters were obtained from the clustering process, all images were then represented in their BoW form. 
After applying frequent itemset mining we observed images contained in these frequent itemsets. We found the value of $k$ to work well between 35,000 and 50,000 cluster centers because most of the patterns that emerged, were observed with these two clusters. The minsup threshold was set at $0.025 \%$ and $0.05 \%$ for mining frequent itemsets for all cases because a lower threshold generated too many itemsets while a higher value resulted in very few itemsets as explained in the next section. For rare itemset mining we only used the transaction datasets generated from 35,000 and 50,000 cluster centers. The minFreqSup was chosen to be $0.04 \%, 0.05 \%$ and $0.06 \%$ and minRareSup was selected as $0.002 \%, 0.004 \%$ and $0.006 \%$, which appeared to be high enough to differentiate noise from a real rare occurrence. The association rules were also generated from these itemsets and only rules with confidence $\geq 0.9$ and lift $\geq 1.0$ were considered interesting.

Here, it is worth noting that although we generate association rules for both mining processes we did not use them for defining relationships among items or image words. Rather we used them for selecting interesting itemsets to test the hypothesis that interesting semantic features will emerge from a large collection of data.

\section{A. Frequent Patterns and Generated Rules}

The mining process generated a large number of itemsets e.g in case of 35,000 clusters up to 1 million itemsets were generated as shown in Table I. Because of this large number it was not possible for us to view images generated by all these itemsets and then decide whether it results in interesting information or not. Instead, we generated association rules with a very high confidence value and used it to prune uninteresting itemsets. Only those itemsets were chosen for viewing their associated images for which there existed a rule that satisfied this confidence criteria. Doing this greatly reduced the total number of itemsets to be viewed. But still in some cases the remaining number of itemsets was very high leaving us with no other option than to randomly sample itemsets for viewing. The sampling was done by keeping the same proportion of itemsets to be examined from varying itemset lengths. On observing images containing these itemsets we found some interesting patterns showing global behavior of these images. From the total itemsets resulted from each mining process approximately 200 itemsets were viewed and each of these were manually categorised into six semantic categories.

The semantic categories were: stripes or parallel lines, dots and checks, bright dots, single lines, intersections and frames as shown in Figure 2. For example all images containing a "dots and checks" pattern had the same semantic concept identified by the itemset (a set of SIFT features in the image) as shown in Figure 2(b). The red marks are the features associated with items (words) in this itemset. Images in each category of Figure 2 were chosen randomly from all the images containing that itemset.

Apart from these patterns, we observed some other patterns showing different behaviors e.g. there were some itemsets
Table I

NUMBER OF FREQUENT ITEMSETS GENERATED AGAINST $k$ CLUSTER CENTERS USING TWO DIFFERENT MIN.SUPPORT THRESHOLDS. THE MINING PROCESS WAS ABORTED WHEN THE GENERATED FILE SIZE REACHED TO $10 \mathrm{~GB}$.

\begin{tabular}{|c|c|c|c|c|c|}
\hline \multirow{2}{*}{ MinSup } & \multicolumn{5}{|c|}{ Total number of itemsets for different cluster centers } \\
\cline { 2 - 6 } & 5,000 & 15,000 & 35,000 & 50,000 & 75,000 \\
\hline $0.025 \%$ & aborted & aborted & 988,354 & 427,398 & 86,203 \\
\hline $0.05 \%$ & aborted & $1,085,926$ & 32,852 & 14,754 & 3,129 \\
\hline
\end{tabular}

Table II

ASSOCIATION RULES GENERATED FROM FREQUENT ITEMSETS FOR TWO VALUE OF CLUSTER CENTERS AND TWO MIN.SUPPORT THRESHOLDS.

\begin{tabular}{|l|l|l|}
\hline \multirow{2}{*}{ MinSup } & \multicolumn{2}{|c|}{ Total number of rules for different cluster centers } \\
\cline { 2 - 3 } & 35,000 & 50,000 \\
\hline $0.025 \%$ & 71,645 & 2,598 \\
\hline $0.05 \%$ & 752 & 556 \\
\hline
\end{tabular}

behaving like an efficient text detector while others resulted in images having either black frames, double frames or hollow circles. But for these itemsets there was also an overlap of images from one or more different semantic patterns e.g the text generating pattern contained images of stripes as well. So we did not categorize them as a separate class rather kept them in one of these 6 categories. Figures 2(a) and 2(f) show some images containing text and different types of framed images. These images were classified into the main categories of stripes and frames respectively. It is also interesting to note that with a different number of clusters not all of these patterns were identified which clearly demonstrates the effect of number of clusters on co-occurrence among features. For the case of 50,000 clusters with minSup threshold of $0.025 \%$ we only observed patterns a,b,c and $\mathrm{f}$ as depicted in Figure 2.

From Table I we can also see the effect of number of clusters on total number of itemsets. Fewer cluster centers generated too many co-occurrences and hence too many itemsets. In some cases we aborted the mining either because of too much time spent by the mining process or the generated file size was too large. A higher value of $k$ on the other hand generated fewer co-occurrences and hence considerably fewer itemsets. As neither too many or too few co-occurrences were desirable, the values of $k$ of 35,000 and 50,000 appeared reasonable.

We observed that with minSup of $0.05 \%$ the number of rules generated was much less with both number of clusters. When viewing images generated by these itemsets we only observed three categories. Patterns a, b and f from Figure 2 were detected for 35,000 clusters while for 50,000 clusters the detected patterns were $\mathrm{a}, \mathrm{b}$ and $\mathrm{c}$. We found that because of a higher confidence (i.e 90\%) most of the itemsets belonging to other categories were pruned. The other patterns were detected when this threshold was reduced to $60 \%$.

For the selection of interesting itemsets we extracted association rules only from itemsets generated from 35,000 and 50,000 cluster centers. Table II depicts the total rules generated against each number of cluster centers. 

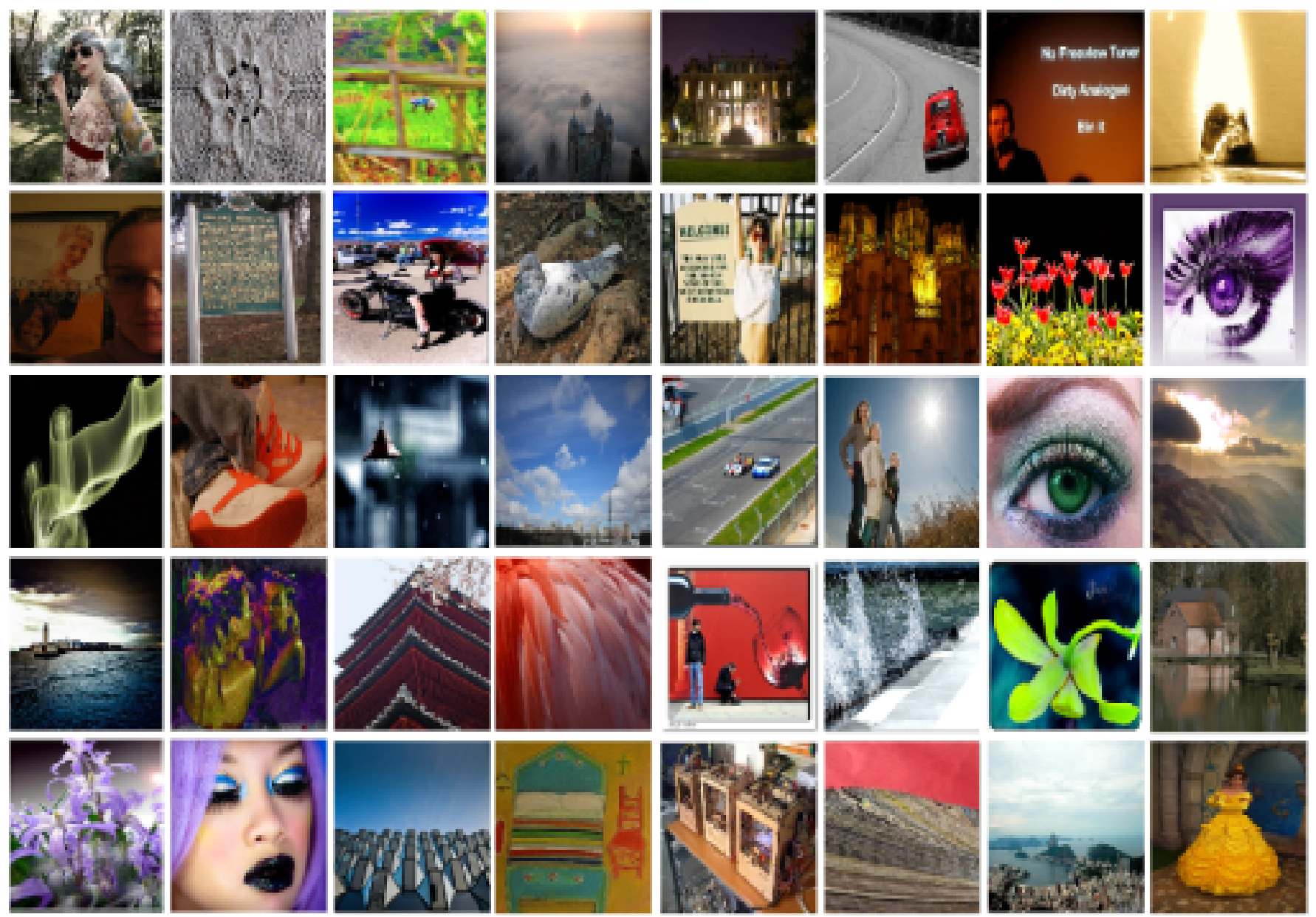

Figure 1. Some images from MIRFLICKR [32] 1 million images collection.

\section{B. Rare Patterns and Generated Rules}

Similar to the case of frequent itemsets, rare itemset mining also generated a large number of itemsets as shown in Table III. Here we also chose 35,000 and 50,000 cluster centers to mine rare items. Association rules were generated from these rare itemsets using the same support, confidence and lift thresholds as defined before. Unlike the frequent itemset case where we observed global patterns, with rare itemsets we hoped to get rare co-occurrences of features or rare patterns. We were interested in co-occurrence that appeared rarely but had a very high confidence. Discovering a rare pattern that a frequent itemset miner might have skipped would be very interesting. From Table IV we can see that for almost all the thresholds the number of generated rules were very high so we randomly sampled rules to select their itemsets. On viewing images generated by these itemsets we discovered that unlike the case of frequent itemsets, here we only found one pattern i.e dots and checks as shown in Figure 3. To further see the effect of number of rare items in the itemsets, the displayed images are ranked by the number of rare items in them. An image with ' 4 ' rare items being the top ranked and ' 1 ' rare item being the lowest ranked. We did not see any itemset
Table III

NUMBER OF RARE ITEMSETS GENERATED FOR 35,000 AND 50,000 CLUSTERS CENTERS AGAINST 3 DIFFERENT VALUES OF minRareSup AND minFreqSup THRESHOLDS. THE MINING PROCESS WAS ABORTED WHEN THE GENERATED FILE SIZE REACHED TO 10GB.

\begin{tabular}{|c|c|c|c|c|}
\hline \multirow{3}{*}{ Clusters } & \multirow{3}{*}{ MinFreqSup } & \multicolumn{3}{|c|}{ MinRareSup } \\
\cline { 3 - 5 } 35,000 & $0.04 \%(200)$ & 312 & 8 & 4 \\
\cline { 2 - 5 } & $0.05 \%(250)$ & aborted & 34,212 & 1,358 \\
\cline { 2 - 5 } & $0.06 \%(300)$ & aborted & $111,101,663$ & $1,771,316$ \\
\hline \multirow{3}{*}{50,000} & $0.04 \%(200)$ & aborted & $25,503,958$ & 395,675 \\
\cline { 2 - 5 } & $0.05 \%(250)$ & aborted & $95,198,773$ & $1,315,304$ \\
\cline { 2 - 5 } & $0.06 \%(300)$ & aborted & aborted & $3,416,042$ \\
\hline
\end{tabular}

containing more than 4 rare items in them. We do not have a good explanation of why only one semantic category was evident for rare itemsets.

\section{CONCLUSION}

In this paper we discussed a method for mining interesting patterns from a large collection of images in an unsupervised scenario. Emerging from these images were 6 semantic categories: stripes, dots, lines, bright dots, intersections and 
Table IV

NUMBER OF ASSOCIATION RULES GENERATED FOR RARE ITEMSETS FOR 35,000 AND 50,000 CLUSTER CENTERS. TWO minRareSup THRESHOLDS AND THREE DIFFERENT minFreqSup WERE USED. THE EMPTY CELL SHOWS THAT NO RULES WERE FOUND BECAUSE OF VERY LESS NUMBER OF ITEMSETS.

\begin{tabular}{|c|c|c|c|c|}
\hline \multirow{2}{*}{ MinFreqSup } & \multicolumn{2}{|c|}{35,000} & \multicolumn{2}{c|}{50,000} \\
\cline { 2 - 5 } & $0.004 \%$ & $0.006 \%$ & $0.004 \%$ & $0.006 \%$ \\
\hline $0.04 \%$ & - & - & 49,515 & 52,243 \\
\hline $0.05 \%$ & 1,247 & 215 & 11,758 & 8,253 \\
\hline $0.06 \%$ & 9,616 & 14,120 & 26,121 & 78,417 \\
\hline
\end{tabular}

frames. We had hoped that some notion of "object-ness" might have emerged from the data, but without using spatial information of the features, this hope was forlorn. Instead, non-local semantic features emerged. An obvious extension is to investigate the effect of also using spatial information when generating interesting rules. Further work on determining whether these bottom-up semantic elements are useful for any of the typical image classification tasks is also warranted.

\section{ACKNOWLEDGMENT}

The authors would like to thank Christian Borgelt and Yun Sing Koh for providing their implementation code for Eclat and $R p$-Tree algorithms respectively.

\section{REFERENCES}

[1] Q. Le, J. Ngiam, Z. Chen, D. Chia, P. Koh, and A. Ng, "Tiled convolutional neural networks," Advances in Neural Information Processing Systems, vol. 23, 2010.

[2] H. Lee, R. Grosse, R. Ranganath, and A. Ng, "Convolutional deep belief networks for scalable unsupervised learning of hierarchical representations," in Proceedings of the 26th Annual International Conference on Machine Learning. ACM, 2009, pp. 609-616.

[3] Y. LeCun, K. Kavukcuoglu, and C. Farabet, "Convolutional networks and applications in vision," in Circuits and Systems (ISCAS), Proceedings of 2010 IEEE International Symposium on. IEEE, 2010, pp. $253-$ 256.

[4] A. Coates, H. Lee, and A. Ng, "An analysis of single-layer networks in unsupervised feature learning," JMLR Workshop and Conference Proceedings.

[5] J. Deng, W. Dong, R. Socher, L.-J. Li, K. Li, and L. Fei-Fei, "ImageNet: A Large-Scale Hierarchical Image Database," in CVPR09, 2009.

[6] M. Sanderson and P. Clough, "ImageCLEF-Cross Language Image Retrieval Track," http://http://imageclef.org//

[7] D. G. Lowe, "Distinctive image features from scale-invariant keypoints," vol. 60, no. 2, 2004, pp. 91-110.

[8] U. Fayyad, G. Piatetsky-shapiro, and P. Smyth, "From data mining to knowledge discovery in databases," vol. 17, 1996, pp. 37-54.

[9] R. Agrawal and R. Srikant, "Fast algorithms for mining association rules in large databases," in VLDB'94, Proceedings of 20th International Conference on Very Large Data Bases, September 12-15, 1994, Santiago de Chile, Chile, J. B. Bocca, M. Jarke, and C. Zaniolo, Eds. Morgan Kaufmann, 1994, pp. 487-499.

[10] M. J. Zaki, S. Parthasarathy, M. Ogihara, and W. Li, "New algorithms for fast discovery of association rules," in 3rd International Conference on Knowledge Discovery and Data Mining (KDD), Aug 1997.

[11] J. Han, J. Pei, Y. Yin, and R. Mao, "Mining frequent patterns without candidate generation: A frequent-pattern tree approach," vol. 8, no. 1, 2004, pp. 53-87.

[12] R. Agrawal, T. Imielinski, and A. N. Swami, "Mining association rules between sets of items in large databases," in Proceedings of the 1993 ACM SIGMOD International Conference on Management of Data, Washington, D.C., May 26-28, 1993, P. Buneman and S. Jajodia, Eds. ACM Press, 1993, pp. 207-216.
[13] H. Pan, Q. Han, G. Yin, W. Zhang, J. Li, and J. Ni, "A roibased mining method with medical domain knowledge guidance," in Proceedings of the 2008 International Conference on Internet Computing in Science and Engineering, ser. ICICSE '08. Washington, DC, USA: IEEE Computer Society, 2008, pp. 91-97. [Online]. Available: http://dx.doi.org/10.1109/ICICSE.2008.91

[14] J. Martinet and S. Satoh, "A study of intra-modal association rules for visual modality representation," in Content-Based Multimedia Indexing, 2007. CBMI'07. International Workshop on. IEEE, 2007, pp. 344-350.

[15] T. Quack, V. Ferrari, and L. V. Gool, "Gool. video mining with frequent itemset configurations," in In Proc. CIVR. Springer, 2006, pp. 360-369.

[16] H. H. Malik, "Clustering web images using association rules, interestingness measures, and hypergraph partitions," in ICWE 06: Proceedings of the 6th international conference on Web engineering. ACM Press, 2006, pp. 48-55.

[17] C. Ordonez and E. Omiecinski, "Discovering association rules based on image content," in Proceedings of the IEEE Advances in Digital Libraries Conference (ADL'99, 1999, pp. 38-49.

[18] T. Quack, V. Ferrari, B. Leibe, and L. J. V. Gool, "Efficient mining of frequent and distinctive feature configurations," in ICCV, 2007, pp. 1-8.

[19] J. Kleban, X. Xie, and W.-Y. Ma, "Spatial pyramid mining for logo detection in natural scenes," in ICME, 2008, pp. 1077-1080.

[20] A. Gilbert, J. Illingworth, R. Bowden, and G. X. England, "Scale invariant action recognition using compound features mined from dense spatiotemporal corners," in In ECCV, 2008.

[21] H. Bay, T. Tuytelaars, and L. V. Gool, "Surf: Speeded up robust features," in ECCV, 2006, pp. 404-417.

[22] C. Harris and M. Stephens, "A combined corner and edge detector," in Proceedings of the 4th Alvey Vision Conference, 1988, pp. 147-151.

[23] T. Lindeberg, "Detecting salient blob-like image structures and their scales with a scale-space primal sketch: A method for focus-ofattention," vol. 11,1993 , pp. 283-318.

[24] - "Edge detection and ridge detection with automatic scale selection," vol. 30, 1996, pp. 465-470.

[25] D. Nistr and H. Stewnius, "Scalable recognition with a vocabulary tree," in CVPR, 2006, pp. 2161-2168.

[26] J. Sivic and A. Zisserman, "Video Google: A text retrieval approach to object matching in videos," in Proceedings of the International Conference on Computer Vision, vol. 2, Oct. 2003, pp. 1470-1477. [Online]. Available: http://www.robots.ox.ac.uk/ $\sim$ vgg

[27] J. Philbin, O. Chum, M. Isard, J. Sivic, and A. Zisserman, "Object retrieval with large vocabularies and fast spatial matching," in CVPR, 2007.

[28] — "Lost in quantization: Improving particular object retrieval in large scale image databases," in CVPR, 2008.

[29] J. L. Bentley, "Multidimensional binary search trees used for associative searching," vol. 18, no. 9. New York, NY, USA: ACM, Sep. 1975, pp. 509-517. [Online]. Available: http://doi.acm.org/10.1145/361002. 361007

[30] B. Goethals and M. J. Zaki, Eds., FIMI '03, Frequent Itemset Mining Implementations, Proceedings of the ICDM 2003 Workshop on Frequent Itemset Mining Implementations, 19 December 2003, Melbourne, Florida, USA, ser. CEUR Workshop Proceedings, vol. 90. CEURWS.org, 2003.

[31] S. Tsang, Y. Koh, and G. Dobbie, "RP-Tree: rare pattern tree mining," 2011, pp. 277-288.

[32] B. T. Mark J. Huiskes and M. S. Lew, "New trends and ideas in visual concept detection: The mir flickr retrieval evaluation initiative," in MIR '10: Proceedings of the 2010 ACM International Conference on Multimedia Information Retrieval. New York, NY, USA: ACM, 2010, pp. 527-536.

[33] C. Borgelt, "Efficient implementations of apriori and eclat," in Proc. 1st IEEE ICDM Workshop on Frequent Item Set Mining Implementations (FIMI 2003, Melbourne, FL). CEUR Workshop Proceedings 90, 2003, p. 90 . 

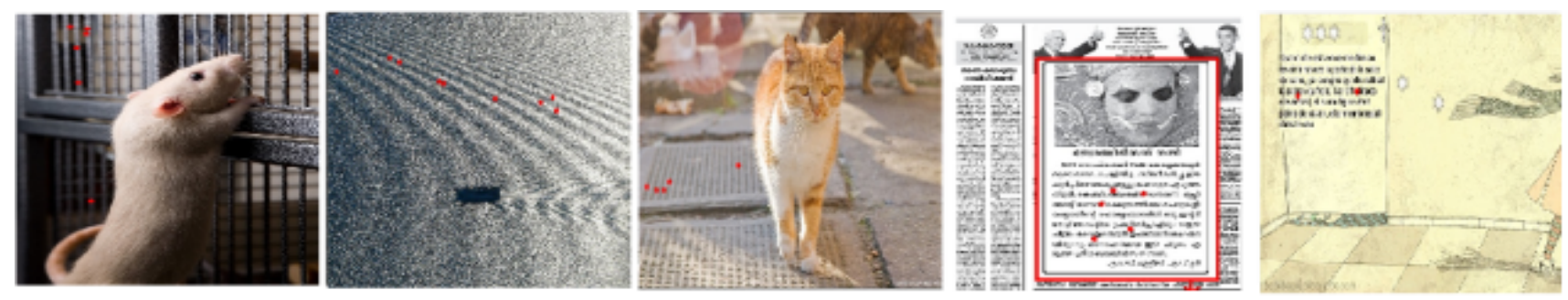

(a) Stripes and parallel lines
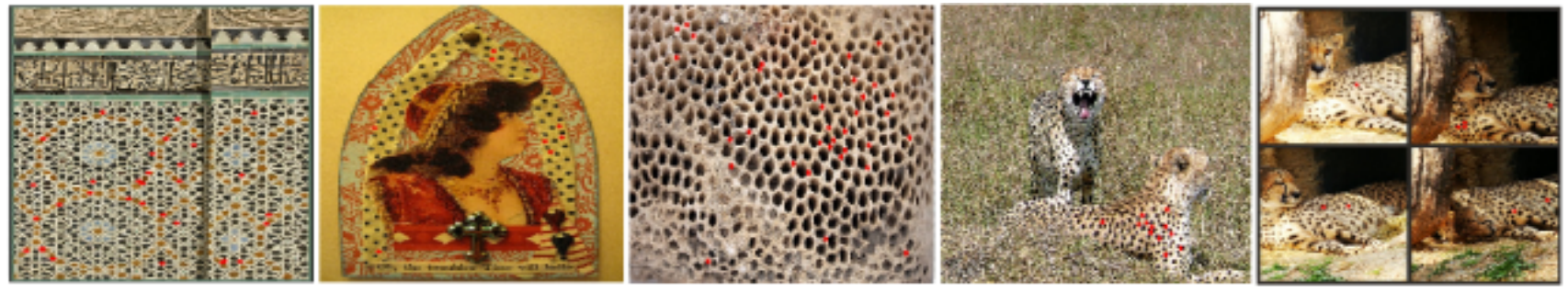

(b) Dots and checks
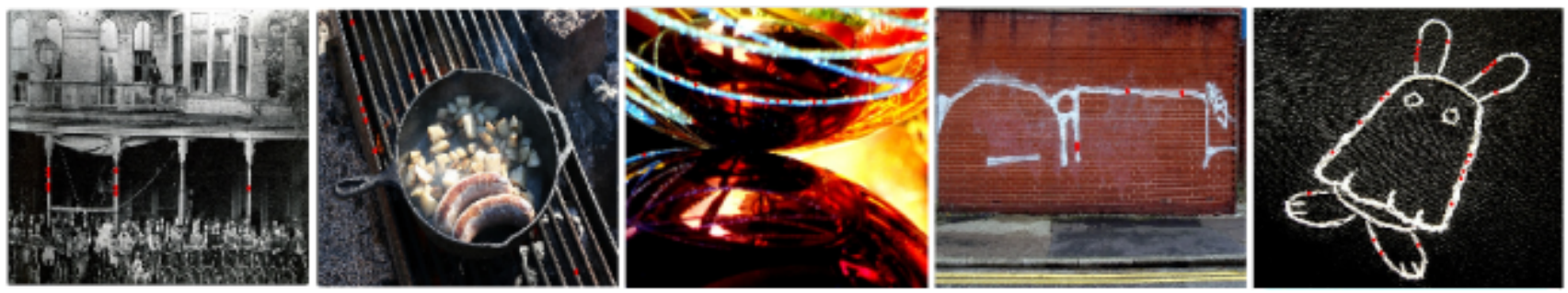

(c) Single lines
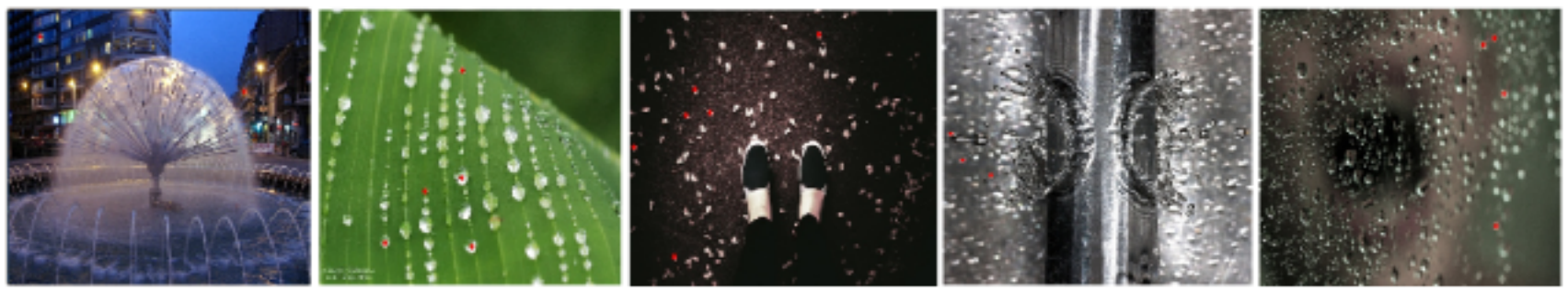

(d) Bright dots
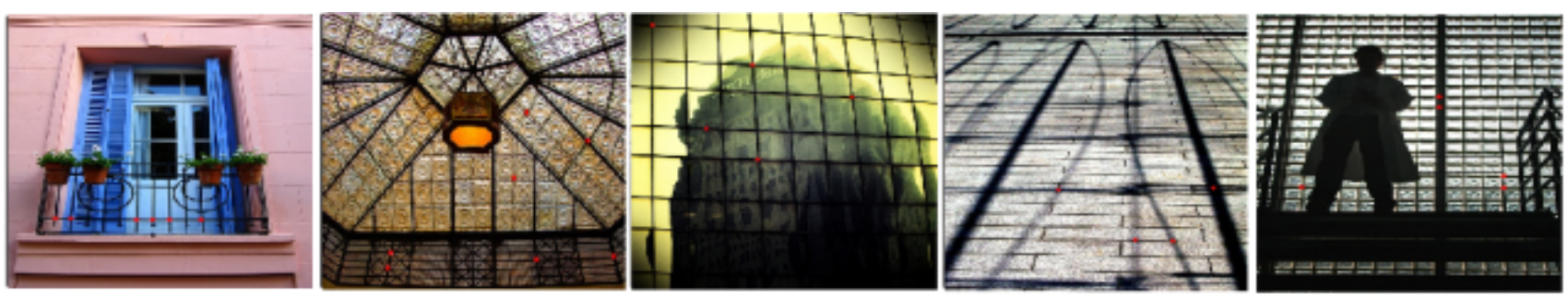

(e) Intersections
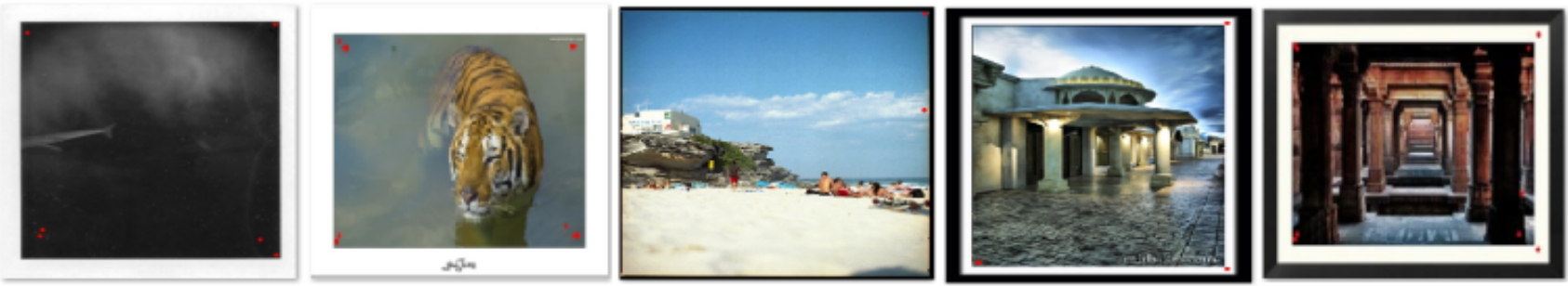

(f) Frames

Figure 2. Itemsets representing different patterns in the images. The elements of the itemset are SIFT keypoint identifiers whose location is specified by a red dot placed on the image. 

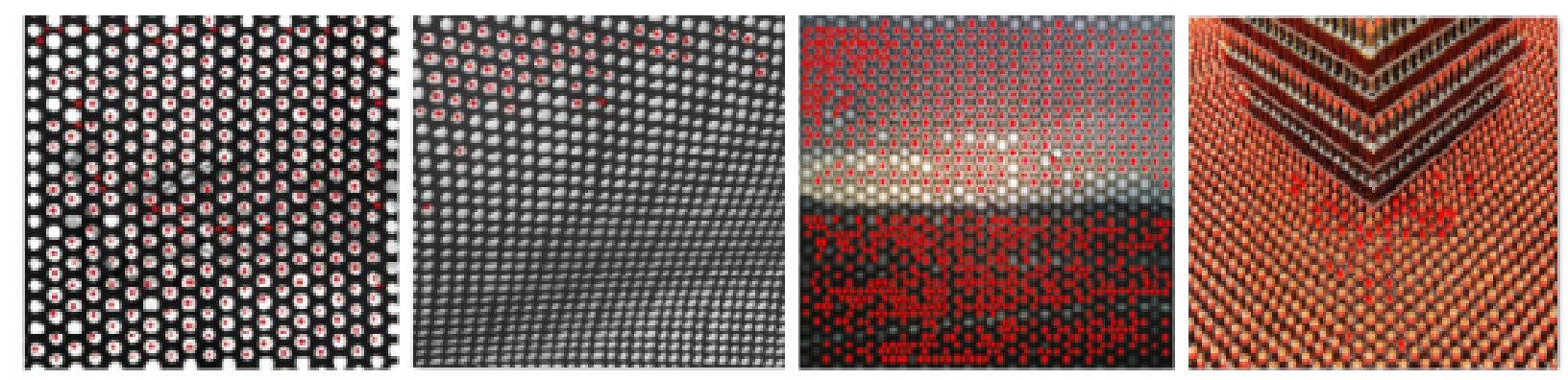

(a) Images containing 4 rare items
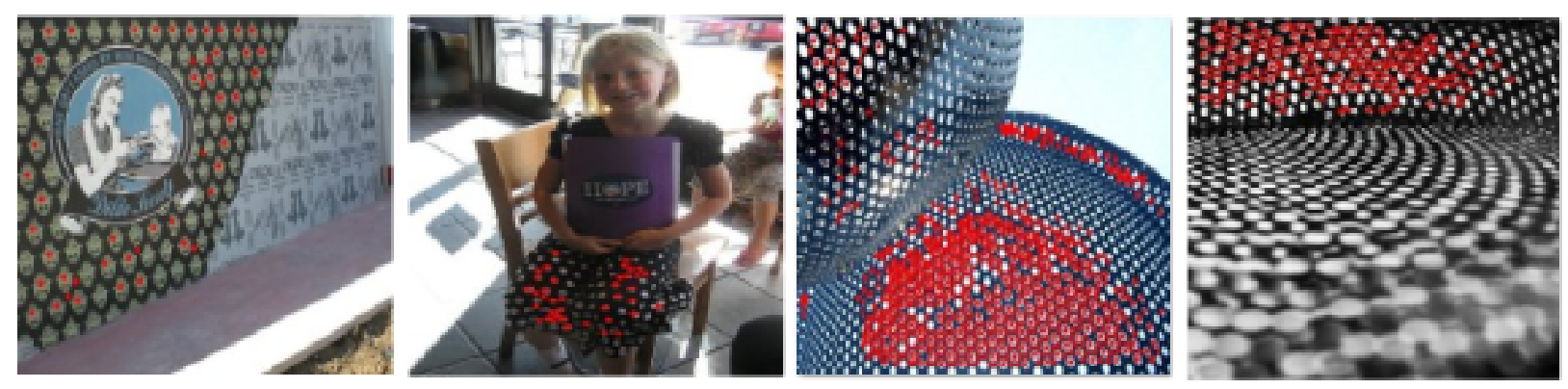

(b) Images containing 3 rare items
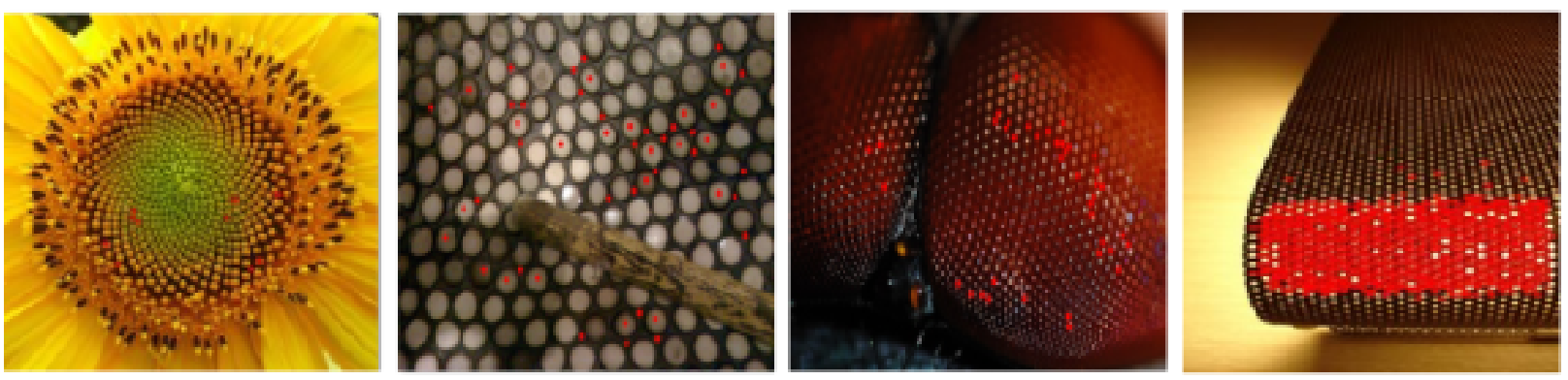

(c) Images containing 2 rare items
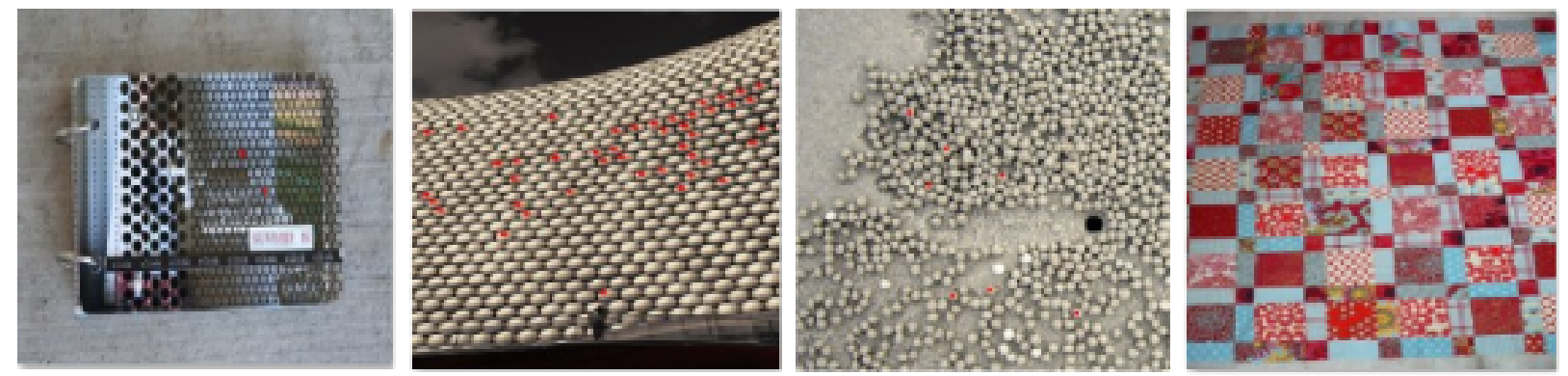

(d) Images containing 1 rare item

Figure 3. Dots and checks: The only semantic pattern observed by rare itemsets mining 